Oriolus Revista Científica, ISSN: 2215-6100.1 (1). Julio, 2020, pp 39-46

Rodríguez González

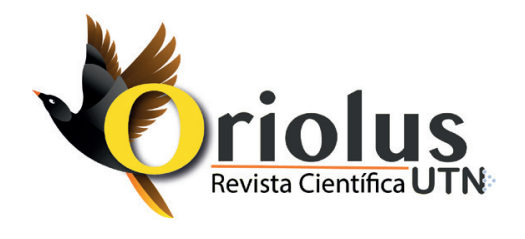

Oriolus Revista Científica

ISSN: 2215-6100

revista.cientifica.oriolus@utn.ac.cr

Universidad Técnica Nacional

\title{
Estudio de crecimiento y de producción de frutos de Bactris guineensis (güiscoyol) en Sistemas Agroforestales como potencial de desarrollo en la Región Chorotega
}

\section{Study of growth and fruit production of Bactris guineensis (güiscoyol) in agroforestry systems as a potential for development in the Chorotega region}

Gabriela Rodríguez González

Estudio de crecimiento y de producción de frutos de Bactris guineensis (güiscoyol) en Sistemas Agroforestales como potencial de desarrollo en la Región Chorotega

Oriolus Revista Científica, Vol.1, N. ${ }^{\circ} 1,2020$

Universidad Técnica Nacional

Disponible en: http://revistas.utn.ac.cr/index.php/oriolus

Bibliografía:

González Rodríguez, Gabriela. Estudio de crecimiento y de producción de frutos de Bactris guineensis (güiscoyol) en Sistemas Agroforestales como potencial de desarrollo en la Región Chorotega. Oriolus Revista Científica, 2020: 39-46. 
Oriolus Revista Científica, ISSN: 2215-6100.1 (1). Julio, 2020, pp 39-46

Rodríguez González

\title{
Estudio de crecimiento y de producción de frutos de
} Bactris guineensis (güiscoyol) en Sistemas

\section{Agroforestales como potencial de desarrollo en la Región Chorotega}

\author{
Study of growth and fruit production of Bactris guineensis (güiscoyol) in \\ agroforestry systems as a potential for development in the Chorotega region
}

Gabriela Rodríguez González

Universidad Técnica Nacional, Costa Rica

mgrodriguezg@utn.ac.cr

(iD https://orcid.org/0000-0003-2098-4708
Recepción: 29 de abril del 2019

Aprobación: 11 de febrero del 2020

\section{Resumen}

Bactris guineensis es una palmera silvestre con potencial productivo en la agroindustria. En la presente investigación se utilizaron en 100 plantas de B. guineensis con edades de 1 a 2 años despues de transplante a las cuales se sometieron a distintas dosis de fertilizante nitrogenado $(50,100,150$ y $200 \mathrm{~kg} / \mathrm{ha}$ ) con el objetivo conocer la biometría de las plantas y hacer aportes al manejo del cultivo. Como resultado relevante se encontró que la cantidad de nitrógeno aplicado es inversamente proporcional al crecimiento y desarrollo de la palmera. Los mejores resultados se registraron a la dosis de $50 \mathrm{~kg} / \mathrm{ha}$ de Nitrógeno. En relación con el diámetro del tallo el fertilizante nitrogenado fue estimulado en pequeñas dosis (0-50 kg/ha) pero a $100 \mathrm{~kg} / \mathrm{ha}$ se obtuvo una inhibición. En investigaciones de este tipo se debe considerar la utilización de bacterias fijadoras de nitrógeno (BFN) como una alternativa en fertilización.

Palabras claves: Producción, sistemas agroforestales, Bactris guineensis, frutos.

\begin{abstract}
Bactris guineensis is a wild palm with productive potential in agroindustry. In the present researching, 100 plants of B. guineensis with ages from 1 to 2 years after transplantation were treated to different doses of nitrogen $(50,100,150$ and $200 \mathrm{~kg} / \mathrm{ha})$ to know the biometry of the plant and contributions to crop management. We found as relevant result that the amount of nitrogen applied is inversely proportional to the growth and development of the palm tree. The best results were registered at the dose of $50 \mathrm{~kg} / \mathrm{ha}$ of Nitrogen. Additionally, we found that stem diameter was stimulated by nitrogen in small doses (0-50 kg / ha), but at $100 \mathrm{~kg} / \mathrm{ha}$, it was inhibited. Works of this type, we must considered the use of nitrogen-fixing bacteria (BFN) as an alternative in fertilization.
\end{abstract}

Keywords: Production, agroforestry systems, Bactris guineensis, fruits. 
Oriolus Revista Científica, ISSN: 2215-6100.1(1). Julio, 2020, pp 39-46

Rodríguez González

\section{Introducción}

La palma Bactris guineensis pertenece a la familia Aracaceae, misma a la que pertenecen el pejibaye (Bactris gasipaes) y el coyol (Acrocomia aculeata), especies silvestres costarricenses. La uva de monte como se le conoce comúnmente en la Región Chorotega es nativa de Centroamérica, el Norte de Colombia y Venezuela. En Costa Rica crece desde el Pacífico Norte hasta Tivives en el Pacífico Central, a una altitud de 0 a 150 m s. n. m. (Henderson, 2000).

La palma es una planta silvestre que puede llegar a medir hasta cinco metros de altura y desarrolla múltiples tallos espinosos, hojas compuestas, alternas, pero agrupadas al final. Su inflorescencia ocurre en enero, julio y septiembre, y sus frutos se han observado en febrero y agosto. Actualmente, los frutos que desarrolla la palma son comestibles, y son utilizados para la elaboración popular y artesanal de mermeladas, refrescos, vinos y chicha (fermentos) en comunidades rurales de la Región Chorotega. Sin embargo, su consumo es estacional y en bajas cantidades. (Chízmar, 2009).

Algunos productores agropecuarios en la provincia de Guanacaste han considerado esta especie como un obstáculo en condiciones de monocultivo, por ejemplo, el arroz (Oryza sativa), pasto de corta (Digitaria decumbens), caña de azúcar (Saccharum officinarum), entre otros. No obstante, se ha observado al ganado consumiendo sus hojas y frutos verdes, como una fuente de alimento en época seca, eviencia potencial poder llegar a ser un sistema agroforestal (Bermúdez et al., 2009).

Un estudio realizado por el Instituto de Investigaciones Farmacéuticas (INIFAR) de la Universidad de Costa Rica, determinó que B. guineensis es una de las especies autóctonas con mayor cantidad de sustancias antioxidantes en comparación con el arándano (vaccinicum consanguineum) y la mora costarricense (Rubus adenotrichum) (Pérez, 2009).

Adicionalmente, se ha investigado el efecto de tratamientos físicos y químicos sobre la semilla para romper la latencia y promover la germinación, utilizando como técnica la exposición a altas temperaturas y a hormonas de crecimiento. En condiciones de almacenamiento, la semilla presenta un reposo muy marcado, por lo que germina en baja proporción (Bermúdez et al., 2017).

Por otro lado, hay escasa información sobre B. guineensis como cultivo comercial y principalmente de su manejo agronómico, lo que incentiva la realización de la presente investigación, por lo que se pretende evaluar el crecimiento en un sistema agroforestal a distintas dosis de fertilizante inorgánico (nitrógeno)

\section{Materiales y métodos}

\section{Descripción del área de estudio}

El área de estudio se localiza en la Finca Experimental de la Universidad Técnica Nacional (UTN) sede Guanacaste, específicamente en el cantón de Cañas. La finca cuenta con 700,48 hectáreas para fines académicos, productivos y reserva forestal.

Los suelos de la finca en su mayoría son de tipo inceptisol con régimen ústico (Ustepts) con características vérticas. El clima de la zona se caracteriza, principalmente, por presentarse una estación seca y una lluviosa, durante la cual se presenta el $90 \%$ de la lluvia anual.

Esta obra esta bajo una Licencia de Creative Commons Atribución NoComercial-SinDerivar Internacional 
Oriolus Revista Científica, ISSN: 2215-6100.1 (1). Julio, 2020, pp 39-46

Rodríguez González

\section{Producción en almácigos}

Los tratamientos corresponden a plantas silvestres de la finca que llevaron una etapa de semillero antes de ser trasplantados al ensayo. El área del ensayo es de $1600 \mathrm{~m} 2$ del cual se sembraron 100 plantas a una distancia de $4 \times 4$ metros. A continuación, se ilustra en la figura 1:

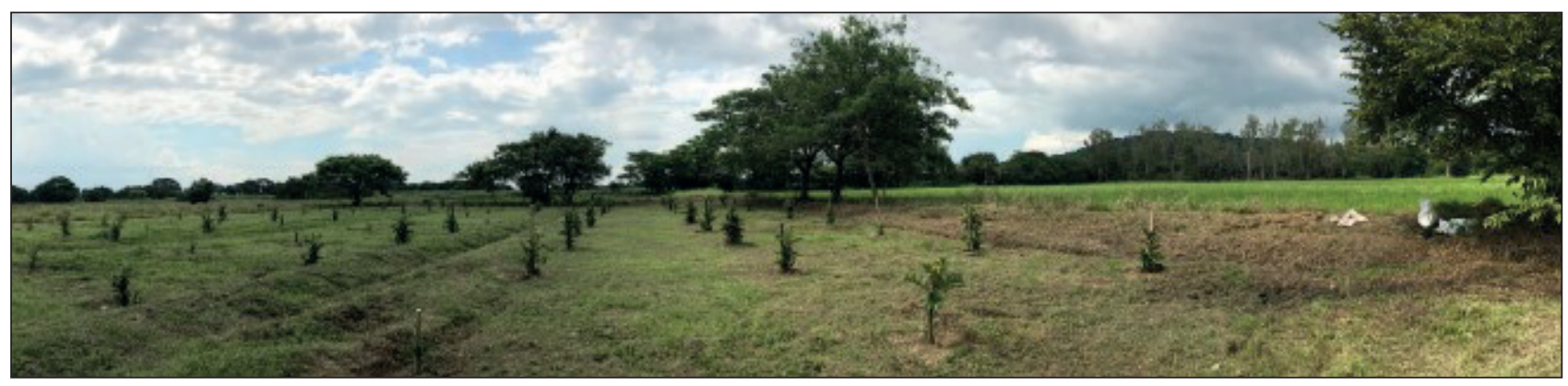

Figura 1. Área de 1600 m2 en la cual se realizaron los trasplantes de B. guineensis. Fuente: elaboración propia.

\section{Diseño experimental}

El diseño experimental consistió en 100 plantas de uva de monte Bactris guineensis, distribuidas al azar en cuatro tratamientos y con una altura promedio de $68 \mathrm{~cm}$, tallo promedio de $15 \mathrm{~mm}$ y un número de hijos promedio de dos por planta.

Los tratamientos fueron aplicados a 20 individuos por tratamiento, con la utilización de distintas dosis de fertilizantes (nitrógeno) y un grupo control. Los cuatro tratamientos T1, T2, T3 y T4 representaron dosis crecientes de fertilizante $(50,100,150$ y $200 \mathrm{~kg} / \mathrm{ha})$, en donde se usó como insumo UREA (46\% N) a una frecuencia de dos aplicaciones por mes y un control sin aplicación. La figura 2 muestra la distribución de los tratamientos en el terreno.

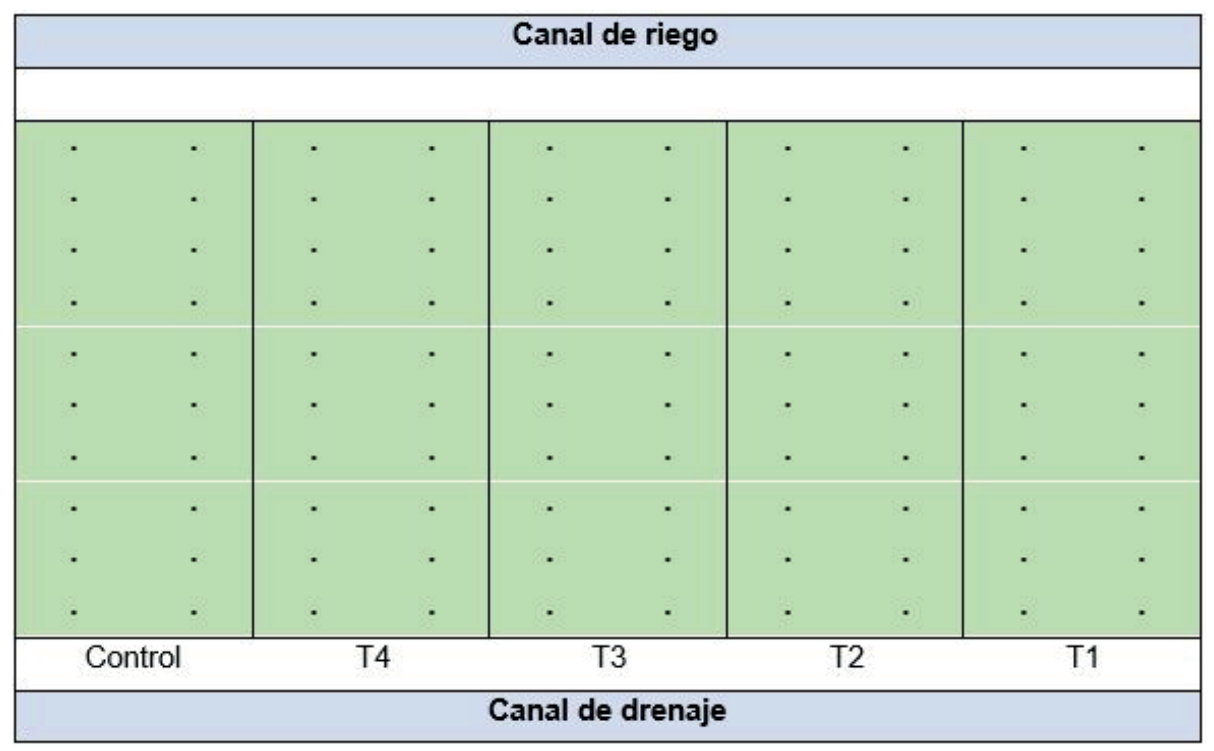

Figura 2. Distribución de los tratamientos en el terreno de estudio. Fuente: elaboración propia (Rodríguez, 2020). 
Oriolus Revista Científica, ISSN: 2215-6100.1 (1). Julio, 2020, pp 39-46

Rodríguez González

\section{Manejo agronómico del cultivo}

En la parcela se contó con riego por gravedad para la época de sequía. Además, se aprovechó la paja de arroz como cobertura muerta al ser incorporada en la circunferencia de la palma y, adicionalmente, se asoció con una leguminosa (Mucuna pruriens) con el fin de realizar un manejo sostenible de las arvenses. Tambien, se colocó un tutor forestal: madero negro (Gliricida sepium) como soporte inicial al cultivo.

\section{Variables biométricas}

Se definieron las siguientes variables biometrciasde crecimiento: altura, diámetro del tallo y número de hijos.

\section{Descriptores de evaluación}

Se anotaron las observaciones para la producción de frutos en las siguientes categorías:

a) Emergencia de la inflorescencia.

b) Presencia de frutos color verde.

c) Frutos color negro-púrpura.

\section{Análisis estadístico previo}

Se compararon los promedios de los datos provenientes de las variables definidas. Las cuales fueron graficadas según muestreos.

\section{Resultados y discusión}

Los datos iniciales arrojaron un promedio de $68 \mathrm{~cm}$ de altura, $15 \mathrm{~mm}$ de diámetro del tallo y un número de dos hijos por planta. Los cuatro muestreos posteriores a las fertilizaciones presentaron un crecimiento del tallo superior en el tratamiento de $50 \mathrm{~kg} / \mathrm{ha}$ y $0 \mathrm{~kg} / \mathrm{ha}$. Se determinó el diámetro del tallo con valores de $16 \mathrm{~mm}$ en los tratamientos T1, T2, T4 y control, situación diferente con el T3 que presentó un diámetro promedio de $12 \mathrm{~mm}$.

El crecimiento de B. guineensis prevalece a nivel de altura más que en el grosor del tallo, como reflejan los resultados obtenidos en la figura 3. A la vez la influencia del fertilizante nitrogenado estimula el crecimiento de la planta en pequeñas dosis, sin embargo, en dosis superiores de $100 \mathrm{~kg} / \mathrm{ha} \mathrm{se}$ obtuvo una inhibición del crecimiento. 
Oriolus Revista Científica, ISSN: 2215-6100.1 (1). Julio, 2020, pp 39-46

Rodríguez González
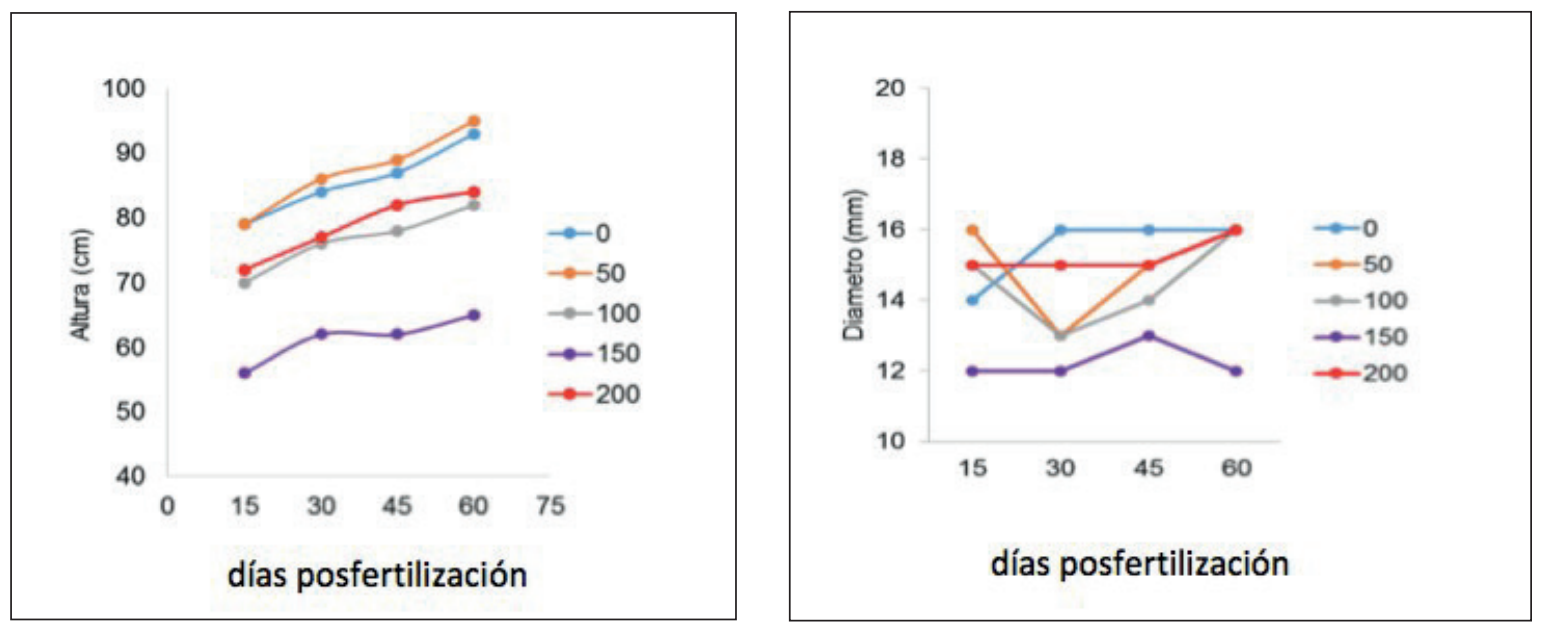

Figura 3. Estimaciones biométricas de B. guineensis. A) Altura y B) Diámetro. Fuente: elaboración propia (Rodríguez, 2020).

Estudios realizados en cultivos provenientes de la misma familia (Aracaceae) han encontrado bacterias fijadoras de nitrógeno en las raíces. Las cuales producen enzimas que toman el nitrógeno de la atmósfera y con los azúcares que obtienen de la planta, fijan el nitrógeno dentro de la biomasa bacteriana para posteriormente trasladaralo a la planta. Por lo tanto, es conocido que el efecto del fertilizante influye en la funcionalidad de estos microorganismos (Shrestha et al., 2009).

Al cuantificar el número de hijos o macollas, se obtuvo un promedio de cuatro hijos en el tratamiento control y en T1, mientrás que los restantes presentaron un promedio de tres hijos por planta. La tabla 1 resume la infromación.

Tabla 1. Producción de hijos de cada tratamiento después de las fertilizaciones.

\begin{tabular}{ccccccc|}
\hline Días Posfertilización & $0 \mathrm{~kg} / \mathrm{ha}$ & $50 \mathrm{~kg} / \mathrm{ha}$ & $100 \mathrm{~kg} / \mathrm{ha}$ & $150 \mathrm{~kg} / \mathrm{ha}$ & $200 \mathrm{~kg} / \mathrm{ha}$ \\
\hline 15 & 3 & 2 & 2 & 2 & 2 \\
30 & 3 & 2 & 2 & 2 & 3 \\
45 & 3 & 3 & 2 & 2 & 3 \\
60 & 4 & 4 & 3 & 3 & 3 \\
\hline
\end{tabular}

Fuente: elaboración propia (Rodríguez, 2020).

Al investigar una especie que tiene un ciclo largo de vida es completamente natural observar entre dos a tres tallos en el segundo año de su plantación. De ese modo es necesario realizar la toma de datos durante todo su periodo de desarrollo y relacionar el nitrógeno con su efecto en el tiempo (Galeano, et al., 2009).

En relación con los análisis observacionales y su clasificación en estado reproductivo de B. Guineensis, las tres categorías presentes durante el periodo de evaluación fueron las siguientes:

Esta obra esta bajo una Licencia de Creative Commons Atribución NoComercial-SinDerivar Internacional 
Oriolus Revista Científica, ISSN: 2215-6100.1 (1). Julio, 2020, pp 39-46 Rodríguez González

a) Emergencia de la inflorescencia.

b) Presencia de frutos color verde.

c) Ausencia de frutos color negro-púrpura.

En el tratamiento 1 (50 kg/ha) se observó tres plantas en categoría A, mientras que para el control se observó una en categoría A y dos en B. Para ampliar la descripción ver figura 4 .
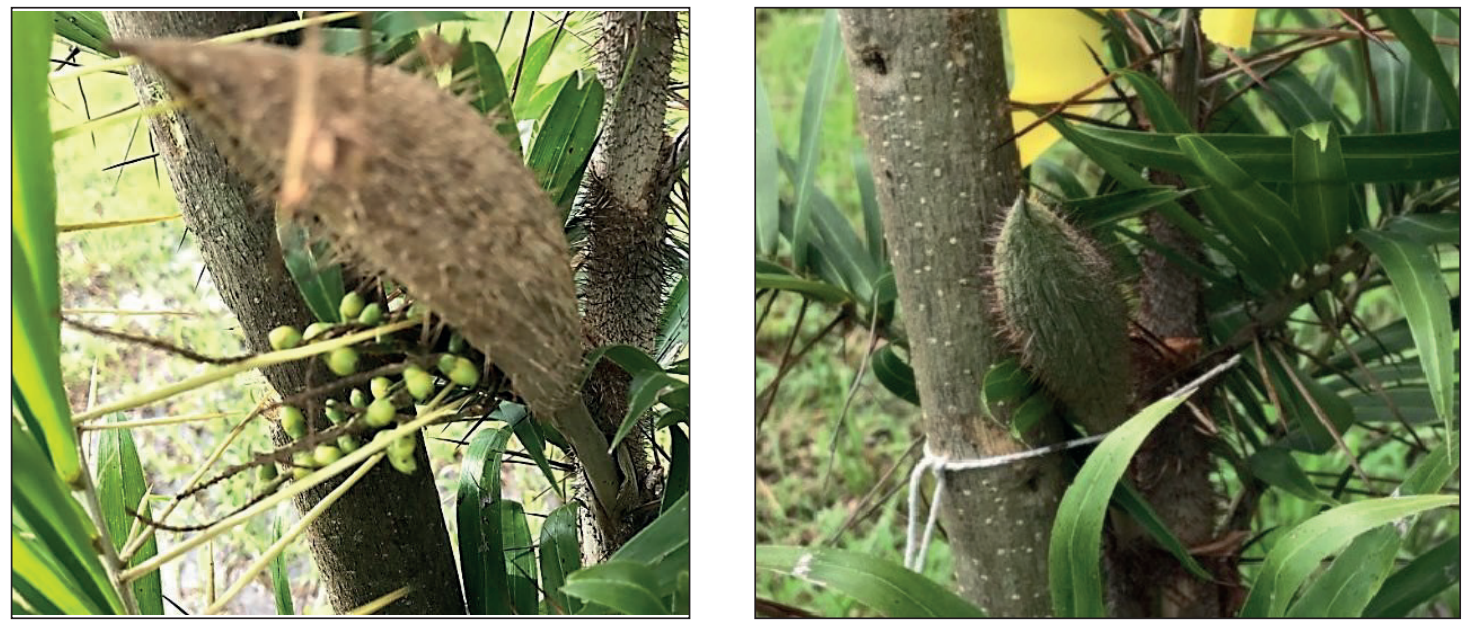

Figura 4. Emergencia de la inflorescencia y presencia de frutos verdes. Fuente: elaboración propia (Rodríguez, 2020).

Esto indica que los primeros estadios del cultivo no son productivos en cuanto al desarrollo de frutos maduros. Se ha observado en plantas silvestres en la provincia de Guanacaste frutos maduros para cosecha en el tercer año después de germinado (Bermúdez, et al., 2009).

\section{Conclusiones}

El fertilizante UREA (46\% N) a dosis mayores de $100 \mathrm{~kg} / \mathrm{ha}$ no produjo resultados positivos en los primeros estadios de crecimientos de B. Guineensis. No obstante, se debe considerar la eficiencia de la aplicación para próximas investigaciones y su relación con los microorganismos presentes en el suelo, principalmente, las bacterias fijadoras de nitrógeno (BFN).

Al relacionar el número de hijos/macollas por planta ninguno de los tratamientos supera al control. Se deduce que se debe a la temprana edad del cultivo o factores ambientales que influyen en la reproducción de la planta.

Durante el periodo del ensayo se observaron las primeras inflorescencias de la palma a los 45 y 60 días después del trasplante, lo que permitió conocer con mayor exactitud el inicio del ciclo reproductivo y su potencial de producción. No obstante, es importante realizar un seguimiento a toda la fenología del cultivo. 
Oriolus Revista Científica, ISSN: 2215-6100.1 (1). Julio, 2020, pp 39-46

Rodríguez González

\section{Bibliografía}

Bermúdez, G. y Montero, W. Estudio sobre el comportamiento natural de la palma Bactris guineensis (L) H. E. Moore en las subcuencas del río Tenorio y Cañas, Cuenca baja del río Bebedero, Costa Rica. Escuela de Ciencias, Universidad Nacional. 2009.

Bermúdez, G., Alizaga, R. y Herrera, J. Efecto de tratamientos físicos y químicos sobre la germinación y almacenamiento de la semilla de Bactris guineensis (L.). Revista Forestal Mesoamericana, Kurú. 2017: 45-54.

Chízmar, C. 2009. Plantas comestibles de Centroamérica. Santo Domingo de Heredia: INBio.

Galeano, G. Bernal, R. y Estupiñán, A. Biología y dinámica poblacional del corozo de lata (Bactris guineensis: Aracacea) en el Caribe Colombiano. Universidad Nacional de Colombia. 2015.

Henderson, A. Bactris (Palmae). Flora Neotropica, 2000: 1-181.

Rojano, B., Zapata, I. y Cortes, F. Estabilidad de antocianinas y valores de capacidad de absorbancia de radicalesoxígeno (ORAC) de extractos acuosos de corozo (Bactris guineensis). Revista Cubana de Plantas Medicinales. 2012: 1028-4796.

Osorio, C., Acevedo, B., Hillebrand, S., Carriazo, J., Winterhalter, P., y Morales, A. Microencapsulation by spray-drying of anthocyanin pigments from Corozo (Bactris guineensis) fruit. Agric Food Chem. 2010: 6977-85.

Pérez, A. Los productos derivados de frutas: fuentes de antioxidantes. CITA/UCR. San José, Costa Rica. 2009.

Shrestha, A., Toyota, K., Nakano, Y., Okazaki, M., Quevedo, M., Loreto, A., Mariscal, A., y Abayon, E. Free-living nitrogen-fixing bacteria are present in the sago palm. Tokyo University of Agriculture and Technology, 2009: 24-16. 